Research Article

\title{
Experimental Research on the Impact of Ion Exchange and Infiltration on the Microstructure of Rare Earth Orebody
}

\author{
Xiaojun Wang, ${ }^{1,2}$ Yulong Zhuo, ${ }^{1,2}$ Shuqiang Deng, ${ }^{1,2}$ Yongxin Li, ${ }^{1,2}$ Wen Zhong, ${ }^{1,2}$ and \\ Kui Zhao, ${ }^{1,2}$ \\ ${ }^{1}$ Jiangxi Key Laboratory of Mining Engineering, Jiangxi University of Science and Technology, Ganzhou, Jiangxi 341000, China \\ ${ }^{2}$ School of Resources and Environment Engineering, Jiangxi University of Science and Technology, Ganzhou, Jiangxi 341000, \\ China \\ Correspondence should be addressed to Yulong Zhuo; 879408217@qq.com
}

Received 24 June 2017; Accepted 30 October 2017; Published 28 December 2017

Academic Editor: Santiago Garcia-Granda

Copyright $\left({ }_{0} 2017\right.$ Xiaojun Wang et al. This is an open access article distributed under the Creative Commons Attribution License, which permits unrestricted use, distribution, and reproduction in any medium, provided the original work is properly cited.

To detect the evolutional characteristics of pore structure in ore leaching process of ion-type rare earth, this paper analyzes the influence mechanism of ion exchange seepage action on the microstructure of orebody, and an experiment for remodeling rare earth saturated samples and ore leaching was designed. Using nuclear magnetic resonance technology obtains the pore structure $T_{2}$ map of $\mathrm{H}_{2} \mathrm{O}$ and $\left(\mathrm{NH}_{4}\right)_{2} \mathrm{SO}_{4}$ solution in the ore leaching process and inverts and reconstitutes the pore structure distribution image. The results of contrastive analysis experiments indicate that impact factors of the ore leaching process on the microstructure of rare earth orebodies include two aspects: solution seepage and ion exchange. The main factor of pore structure distribution is the ion exchange action, determined by a dual effect. The sole action of solution seepage leads to an increase in pore size, which means that pore size structure is changing from small and medium to macro. Ion exchange gives rise to the movement and restructuring of particles, which results in a decrease in pore sizes. The pore structure changes from loose to compact; in the entire ore leaching process, the ion exchange action advances in a layered shape along the direction of seepage, and the chemical replacement and physical seepage alternately impact the microstructure of the orebodies.

\section{Introduction}

Ion-adsorption rare earth ore is one of the most precious and rare types of ore in the world and is found in the strong mantle rock of shallow ground [1-3]. At present, the in situ leaching mining method is a mature mining technology, which takes advantage of the chemical replacement reaction of leaching solutions in the process of orebody seepage to achieve the recovery of rare earth cations and thus realizes the extraction of rare earth elements [4-6]. The permeability characteristics of the leaching solution in the orebody rely heavily on the distribution of the internal microscopic pore structure. That is, the form and distribution parameters of the microstructure directly influence the diffusion and penetration of the leaching solution in rare earth orebodies. Therefore, research on the chemical replacement process of the leaching solution and the characteristics of orebody microstructures is beneficial for furthering the analysis of the leaching infiltration mechanisms and is of great significance to green extraction and effective recovery of ion-type rare earth ores.

The matrix of rare earth ore is strong, weathered granite, belonging to the same discrete heterogeneous body as ordinary rock and soil media. In recent years, with the wide application of microcomputed tomography technology [7-9], scholars have started to study the evolution and influencing mechanisms of rock and soil media microstructures. Kodali et al. conducted research on the distribution characteristics of microfissures inside copper ore particles, taking advantage of micro-CT [10]. Nosrati et al. applied micro-CT to scan the internal structure of nickel granulation ore and discovered the microstructure distribution law [11]. Yan et al. introduced microparameters to describe the pore characteristics of soil [12]. Cui et al. measured the pore characteristics of loess-adopting ratecontrolled mercury and analyzed the influence of vacuum 


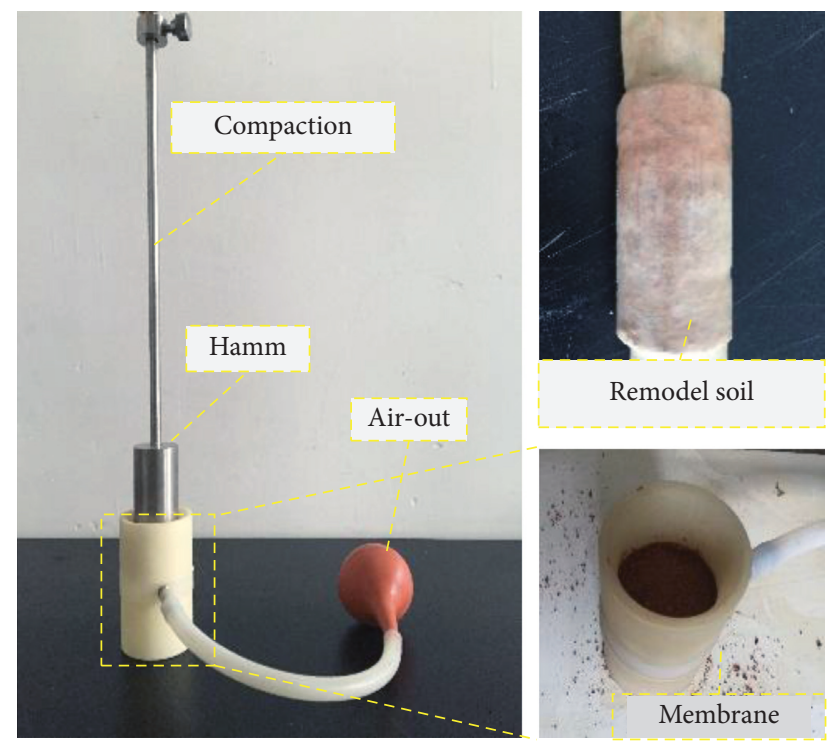

Figure 1: Manufacturing process of remodeling soil specimens.

freeze-drying and drying on the microstructure of loess [13]. $\mathrm{Li}$ et al. studied the change law of pore structures, void ratios, and moisture content in the process of soil discharge and established a corresponding relationship among them [14].

The in situ leaching of ion-type rare earth involves two important processes: ion exchange and solution infiltration. Ion exchange is a chemical replacement process, while solution infiltration is a physical migration process. At present, little research has been carried out to detect whether the coupling effect of these two processes will result in a change in the micropore structure of rare earth orebodies, and useful conclusions have not yet been reached. This paper describes the uses of nuclear magnetic resonance technology to study the microstructure evolutionary mechanisms of rare earth orebodies in the processes of ion exchange and infiltration and provides important research references for the leaching and mining of ion-type rare earth ores.

\section{Experimental Section}

\subsection{Principles of the Experiment}

2.1.1. Chemical Replacement. Rare earth elements are present in strong, weathered granite in the form of ions. $\left(\mathrm{NH}_{4}\right)_{2} \mathrm{SO}_{4}$ solution is usually adopted in the leaching process $[15,16]$. The rare earth cations absorbed by the orebody will be replaced in the leaching solution after contact with $\mathrm{NH}_{4}^{+}$, which has more active chemical properties, as shown in (1). This strong chemical reaction occurs throughout the entire leaching process. Ion exchange involves the adjustment and reconstruction of the mineral microstructures, leading to a change in the crystal structure. The seepage effect of leaching solution also exerts an impact on the microstructure of orebodies:

$$
\begin{aligned}
& 2(\text { Kaolin })^{3-} \cdot \mathrm{RE}^{3+}+3\left(\mathrm{NH}_{4}^{+}\right)_{2} \cdot \mathrm{SO}_{4}^{2-} \\
& =2(\text { Kaolin })^{3-} \cdot\left(\mathrm{NH}_{4}^{+}\right)_{3}+\mathrm{RE}_{2}^{3+}\left(\mathrm{SO}_{4}^{2-}\right)_{3} .
\end{aligned}
$$

TABle 1: Physical parameters of rare earth ore sample.

\begin{tabular}{lcccc}
\hline $\begin{array}{l}\text { Parameter } \\
\text { type }\end{array}$ & $\begin{array}{c}\text { Geometry } \\
\text { dimension }(\mathrm{mm}) \\
\text { Diameter }\end{array}$ & $\begin{array}{c}\text { Density } \\
\left(\mathrm{g} / \mathrm{cm}^{3}\right)\end{array}$ & $\begin{array}{c}\text { Moisture } \\
\text { content }(\%)\end{array}$ \\
\hline Value & 40 & 60 & 1.75 & 15 \\
\hline
\end{tabular}

2.1.2. Test of the Microstructure. Nuclear magnetic resonance (NMR), as a new type of detection technology, has been widely applied in the field of geotechnical engineering [17]. The most important characteristic of this technology is that it can quickly, accurately, and quantitatively measure the microstructure of pores and the pore size distributions while not destroying the structure of rock or soil. Therefore, this is a common detection technology for the analysis of the microstructure evolution of rare earth orebodies in the process of leaching.

The association of the transverse relaxation time, $T_{2}$, and the solid-liquid separation characteristics in the leaching process, as well as the specific surface area of pores, is more evident, which reflects the change in the characteristics of the pore structures in rare earth samples in the leaching process to a certain extent. According to NMR principles, free relaxation and diffusion relaxation can be neglected [18-20], and the transverse relaxation rate can be simplified as

$$
\frac{1}{T_{2}}=\frac{\rho_{2}}{S V}
$$

In (2), $\rho_{2}$ represents the relaxation strength $(\mu \mathrm{m} / \mathrm{ms})$, $S$ represents the pore surface area $\left(\mathrm{cm}^{2}\right)$, and $V$ represents the pore volume $\left(\mathrm{cm}^{3}\right)$. In (2), the ratio between the surface area and volume determines the size of the pores, and the transverse relaxation rate reflects the frequency of proton collisions in the pores. Smaller pore sizes lead to more frequent collisions. Therefore, the transverse relaxation time, $T_{2}$, is directly proportional to the size of the pores. The spectrum analysis curve of $T_{2}$ in the leaching process of a rare earth orebody can be obtained through the detection results, which can then identify the change rule of the pore structures.

2.2. Experimental Method and Process. The sample was acquired through the remodeling of rare earth sample ore from a mine, and the sample size was consistent with the effective detection area of NMR. The diameter and height of the sample were $40 \mathrm{~mm}$ and $60 \mathrm{~mm}$, respectively. In the remodeling process, the consistency of the density of the original soil sample and its moisture content were maintained to the greatest possible extent, as shown in Figure 1. After all phase detection, the rare earth oxide (REO) of the original sample was $0.065 \pm 0.003 \%$, which conforms to the experimental requirements. The physical parameters of remodeling the rare earth sample are shown in Table 1.

To analyze the evolution law of the pore structure of the rare earth sample in the ion exchange process, contrastive analysis experiments were designed. The leaching experiment of remodeling the rare earth sample was conducted on 


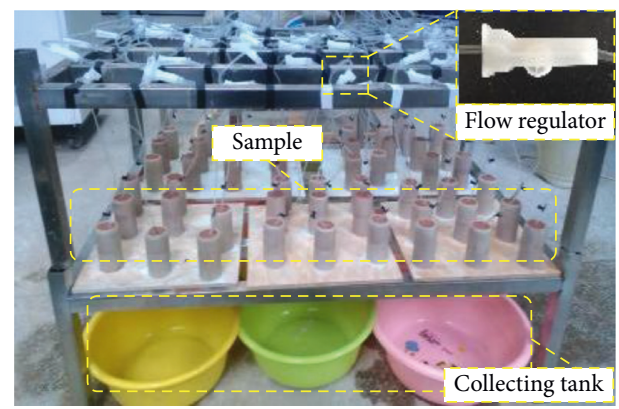

FIGURE 2: Simulation leaching.

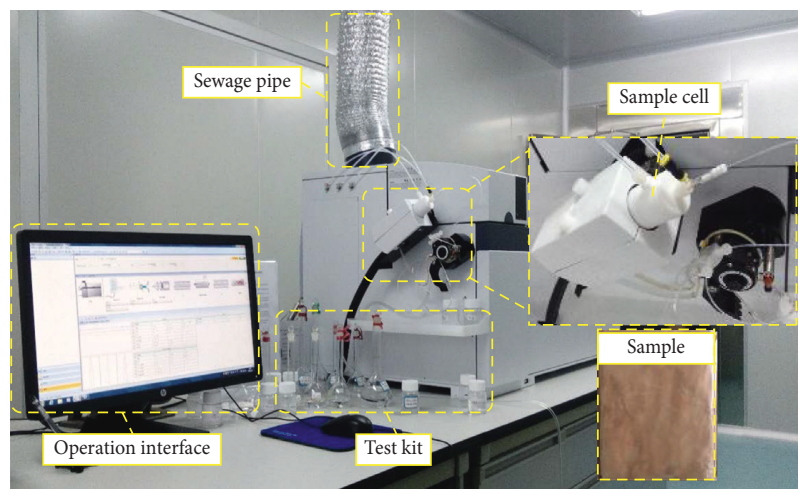

FIGURE 3: Inductively coupled plasma mass spectrometer (ICP-MS).

the simulative leaching experiment setup (the flow rate is $1 \mathrm{~mL} / \mathrm{min}$ ), as shown in Figure 2. The entire leaching experiment was divided into two parts. In the first part, the duration of leaching was six hours, and pure water was selected as the leaching fluid. In this process, a chemical replacement reaction was not involved. In the second part, the duration of leaching was six hours, but a $2.5 \%\left(\mathrm{NH}_{4}\right)_{2} \mathrm{SO}_{4}$ solution, which is commonly used in industrial experiments, was selected as the leaching agent. According to the replacement principle, the second part $(7-12 \mathrm{~h})$ is the ion exchange process. The two parts of the experimental results were contrastively analyzed.

To effectively identify the ion exchange rate of the chemical replacement process in the different leaching phases, all phase detections of REO were conducted every hour during leaching. When conducting the detection, two complete samples were taken and dried, and then the samples were crushed into powder. The REO in a sample was determined using an inductively coupled plasma mass spectrometer (ICP-MS), as shown in Figure 3, which represents the ion exchange process during mineral leaching.

The micropore structure of the sample was measured using a PQ-OO1-type mini-NMR spectrometer, as shown in Figure 4. The permanent magnets possess a magnetic field intensity of $0.52 \mathrm{~T}$ (Tesla). The valid experimental area of the sample was $\phi 60 \mathrm{~mm} \times 60 \mathrm{~mm}$, and the temperature of the permanent magnets was $32 \pm 0.01^{\circ} \mathrm{C}$, ensuring the stability and uniformity of the experimental magnetic field. Two typical samples were obtained before leaching, and the micropore structures of samples were detected every hour,

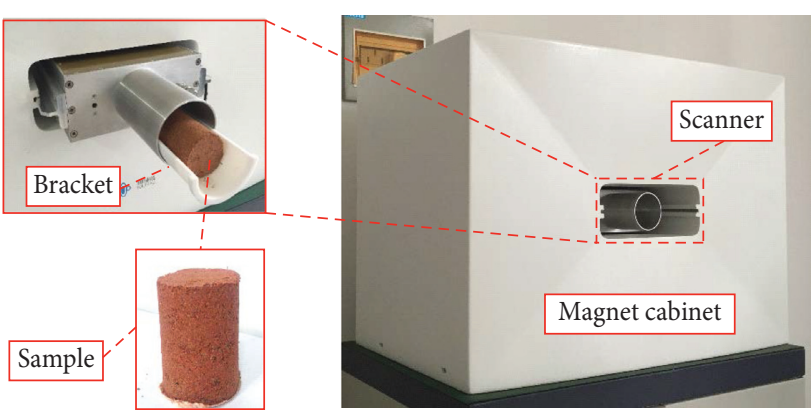

Figure 4: PQ-OO1-type mini-NMR spectrometer.

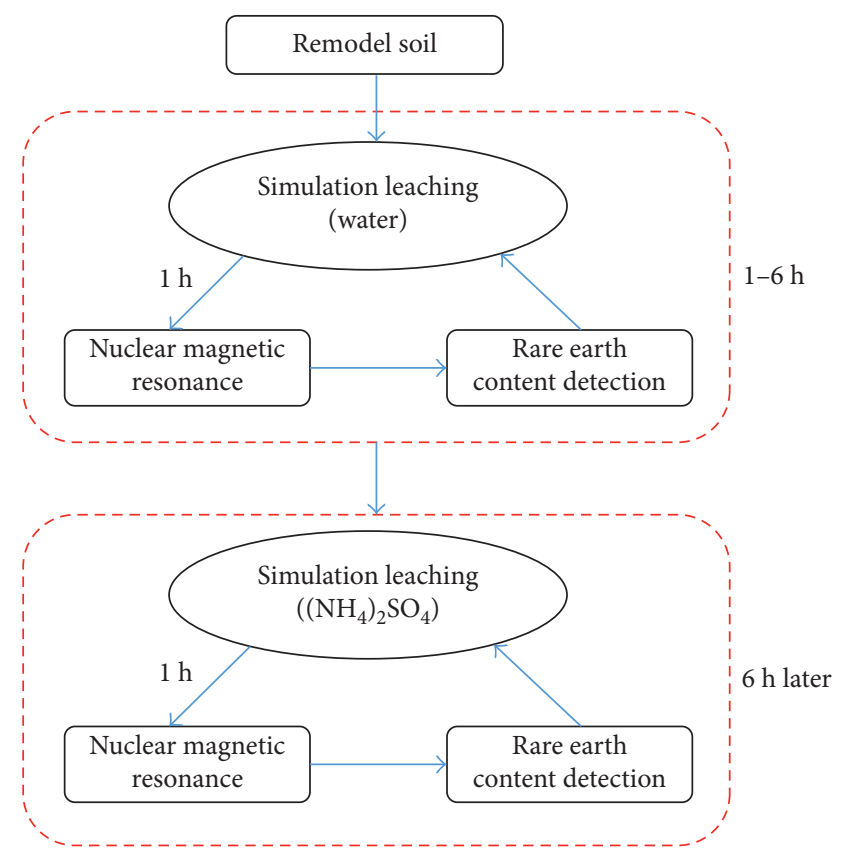

Figure 5: Test process.

based on the interval of leaching. Additionally, each sample was leached for $1 \mathrm{~h}$, and its porosity was determined before continuing the leaching for another hour, using the same sample; the porosity was measured again and the process continued. The entire test process is shown in Figure 5.

\section{Analyses of Experimental Results}

3.1. Change in REO in the Leaching Process. During leaching, the solid sample is chemically analyzed every hour, acquiring the REO in different periods, to analyze each phase of the ion exchange reaction in the leaching process. The experimental results of each period are shown in Table 2.

Combined with the REO changing curve in the leaching experiment process (Figure 6), we know that $\mathrm{H}_{2} \mathrm{O}$ is used as the leaching solution in the first phase. No change occurs in the REO concentrations of the solid samples, with measurements being stable within the margin of error. Thus, it can be deduced that the ion exchange reaction, as shown in (1), does not occur inside the sample and that the rare earth cations are not extracted by the leaching solution when applying $\mathrm{H}_{2} \mathrm{O}$ as the leaching solution. After the first six 
TABLE 2: REO in the leaching process.

\begin{tabular}{lcc}
\hline Leaching solution & Leaching time $/ \mathrm{h}$ & $\mathrm{REO} / 10^{-6} \mathrm{~g} / \mathrm{t}$ \\
\hline & 0 & 649 \\
& 1 & 652 \\
& 2 & 647 \\
$\mathrm{H}_{2} \mathrm{O}$ & 3 & 657 \\
& 4 & 641 \\
& 5 & 639 \\
& 6 & 650 \\
\hline & 7 & 630 \\
& 8 & 600 \\
$\left(\mathrm{NH}_{4}\right)_{2} \mathrm{SO}_{4}(2.5 \%)$ & 9 & 470 \\
& 10 & 290 \\
& 11 & 200 \\
& 12 & 180 \\
& 13 & 178 \\
& 14 & 181 \\
\hline
\end{tabular}

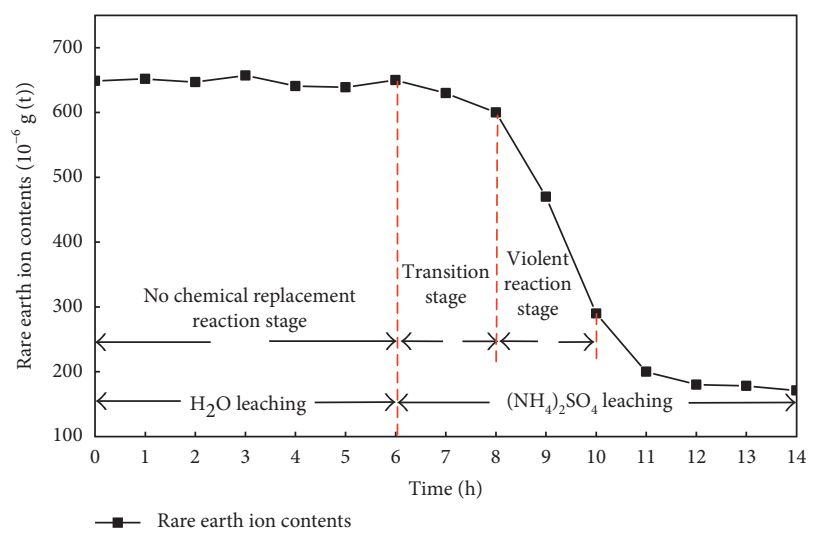

FIGURE 6: REO changing curve in the leaching experiment process.

hours, the leaching solution is replaced by $2.5 \%\left(\mathrm{NH}_{4}\right)_{2} \mathrm{SO}_{4}$ solution. In the following analyses, the REO level gradually declines in the sample. The REO declines slowly between the 6th and the 8th hour, which is mainly due to the fact that leaching solutions containing rare earth cations do not extract in large amounts but remain in the sample matrix. After a prolonged time, the chemical replacement reaction occurs intensively in the sample matrix, and the leaching solution containing rare earth cations continuously extracts. We can see from Figure 6 that the REO in the sample matrix falls sharply between the 8th and 10th hour. Between the 10th and 14th hour, the REO drops to below 200 and the change begins to flatten, which proves that the ion exchange reaction is almost completed in the sample after the 10th hour. It can be deduced that, based on the above analyses, the early phase, between the 0 and 6th hour, belongs to the phase without a chemical replacement reaction; the phase between the 6th and 11th hour belongs to the intensive ion exchange phase; and the ion exchange reaction is completed after the 12th hour.

3.2. Distribution of NMR $T_{2}$. The $T_{2}$ spectrum curve is closely related to the change in the inner pores. The

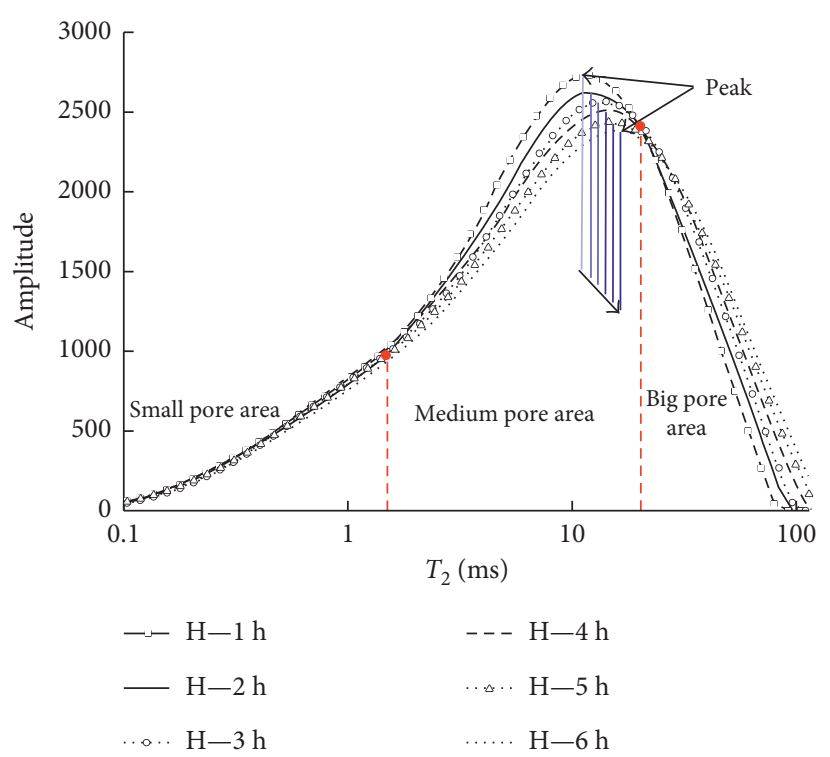

Figure 7: $\mathrm{H}_{2} \mathrm{O}$ leaching (the first phase).

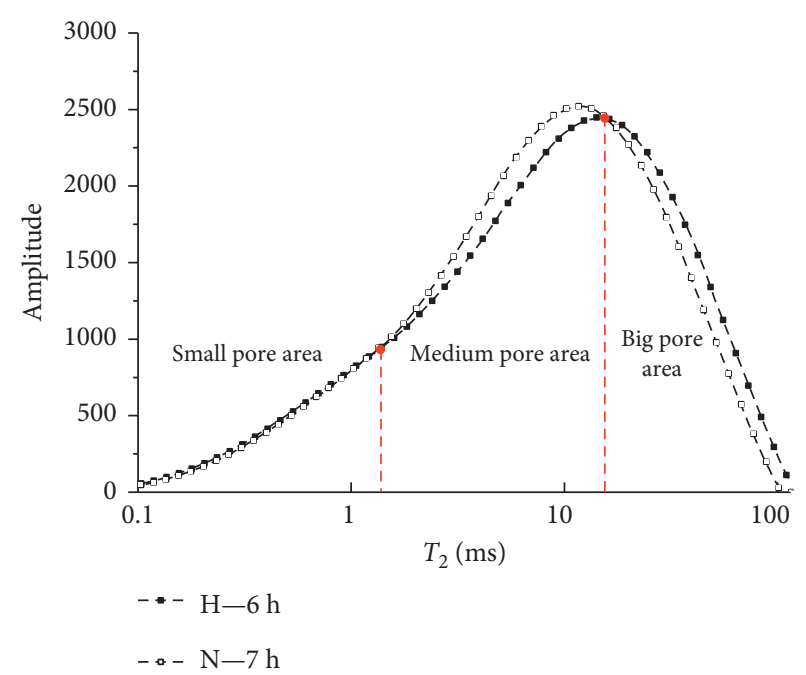

FIgUre 8: Transition phase of $\mathrm{H}_{2} \mathrm{O}$ and $\left(\mathrm{NH}_{4}\right)_{2} \mathrm{SO}_{4}$ (the second phase).

$T_{2}$ spectrum curve can reflect the sizes of pores in the rare earth specimen and the number of pores of different sizes. The size of the $T_{2}$ value is positively related to the pore size. The amplitude represents the number of pores of different sizes.

The entire process in the mineral leaching experiment is divided into three phases. The first phase is $\mathrm{H}_{2} \mathrm{O}$ leaching, the second phase is the transition phase of $\mathrm{H}_{2} \mathrm{O}$ and $\left(\mathrm{NH}_{4}\right)_{2} \mathrm{SO}_{4}$, and the third phase is $\left(\mathrm{NH}_{4}\right)_{2} \mathrm{SO}_{4}$ leaching. The $T_{2}$ spectrum distribution curves of the three phases are shown in Figures 7-9, respectively.

Figure 7 presents the $T_{2}$ spectrum curve of the entire process of $\mathrm{H}_{2} \mathrm{O}$ leaching. We can see from the area formed by the spectrum curve that the inner pore distribution of the remodeled rare earth sample is dominated by pores with medium apertures. Compared with the curve distribution between the 1st and 6th hour, we can see that an obvious 


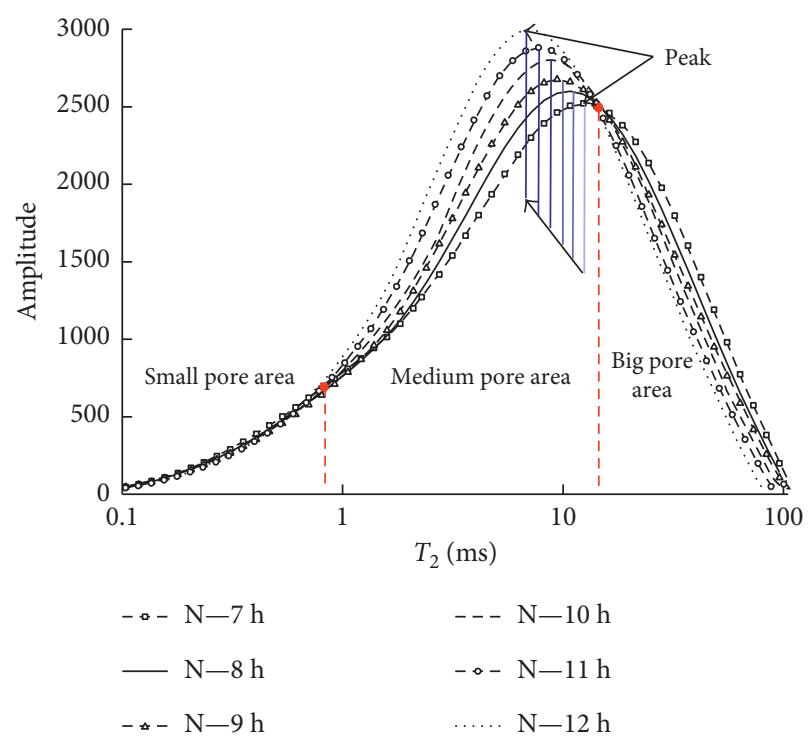

FIGURE 9: $\left(\mathrm{NH}_{4}\right)_{2} \mathrm{SO}_{4}$ leaching (the third phase).

change in the curve spectrum of the pore area with medium apertures has taken place after this prolonged amount of time. A specific manifestation is that the peak value gradually decreases and the crest moves to the right. With the analysis of the pore area with large apertures, we can discover that the large aperture segment of the $T_{2}$ spectrum curve is gradually moving to the right, which explains that the size of the pores with large apertures are also gradually increasing inside the sample in the leaching process. Under the simple seepage action of the leaching fluid, the change in internal microstructure of an ion-type rare earth orebody is that the pores with medium apertures gradually become pores with large apertures; the number of pores with medium apertures decreases, while the number of pores with large apertures increases.

According to the results shown in Figure 8, the leaching fluid is replaced by the $2.5 \%\left(\mathrm{NH}_{4}\right)_{2} \mathrm{SO}_{4}$ solution after pure water leaching for six hours. During the 7 th hour, we can see from the analysis of the leaching results that the change law of the $T_{2}$ spectrum curve is not consistent with that of pure water leaching. The peak value of the $T_{2}$ spectrum curve increases and the crest moves to the left, which is opposite of the previous change law.

We can see from Figure 9 that the peak value of the entire $T_{2}$ spectrum curve increases and the crest moves to the left after leaching by the $\left(\mathrm{NH}_{4}\right)_{2} \mathrm{SO}_{4}$ solution from the 7 th to 12th hour, which explains that the number of pores with medium apertures is increasing and the pores with medium apertures are gradually becoming pores with small apertures after leaching by the $\left(\mathrm{NH}_{4}\right)_{2} \mathrm{SO}_{4}$ solution. We can also see from the pore area of the large apertures in the $T_{2}$ spectrum curve that after a prolonged time, the $T_{2}$ spectrum curve of the large apertures moves to the left, gradually moving toward the pore area of medium apertures. According to the analysis results presented in Figure 6, the period between the 6 th and 12th hour is the ion exchange reaction phase. Not only does the infiltration process exist in this phase but also

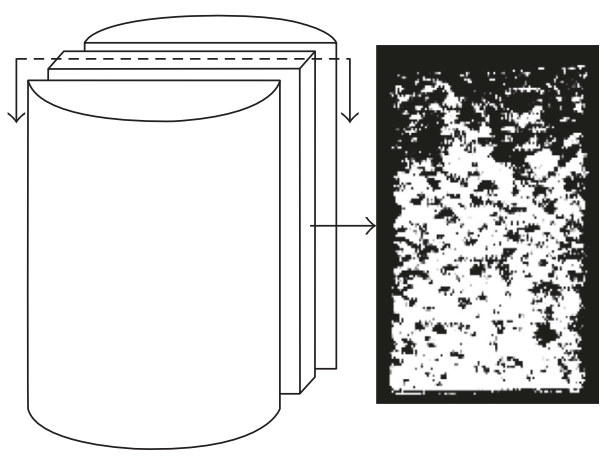

Figure 10: Typical cutting plane.

the chemical replacement process. Comparing Figure 7 with Figure 9, we can see that the main factor influencing the micropore structure of rare earth orebodies is the ion exchange reaction. Therefore, the ion exchange reaction inside the rare earth orebody will induce a decrease in the pore volume inside the orebody. Moreover, the aperture size shows the tendency of changing from large to small overall, and the entire micropore structure of the orebody tends to be more compact.

3.3. Imaging Analysis of Microstructure Evolution. From the analysis presented in Section 2.2, we can see that the ion exchange reaction between the rare earth orebody and the leaching solution changes the structural chain among particles, leading to the recombination of particles among inner pores and giving rise to new changes in the micropore structure. The reconstruction imaging technology of NMR is applied to conduct image reconstruction of the scanning data at regular intervals, obtaining the inversion image of the microstructure. To conduct an intuitive analysis of the micropore structure change law, the inversion image is projected. The leaching solution infiltrates along the axis of the sample, and the typical cutting plane is thus selected in the center of the sample along the generatrix, as shown in Figure 10.

Figures 11 and 12 are two-dimensional images of the cutting plane in the leaching process. Figure 11 shows the phase of pure water leaching, while Figure 12 shows the phase of $2.5 \%\left(\mathrm{NH}_{4}\right)_{2} \mathrm{SO}_{4}$ solution leaching. The bright color shows the area of liquid molecules, which is the area of the pores. The activity of the water molecules in the black area is extremely weak, so this area is considered a solid region.

It can be clearly seen that after a prolonged time, the bright area gradually expands when pure water is used as the leaching solution, which explains why the number of inner pores increases after a prolonged time and why the apertures also increase under the sole action of the seepage effect, which is completely consistent with the analysis results in Section 2.2.

The most important change appears in Figure 12. This image shows the change when the leaching solution is replaced by the $\left(\mathrm{NH}_{4}\right)_{2} \mathrm{SO}_{4}$ solution. We can see from the image of the 7th hour that the bright area decreases slightly in the upper part, and the black area starts to increase. This 


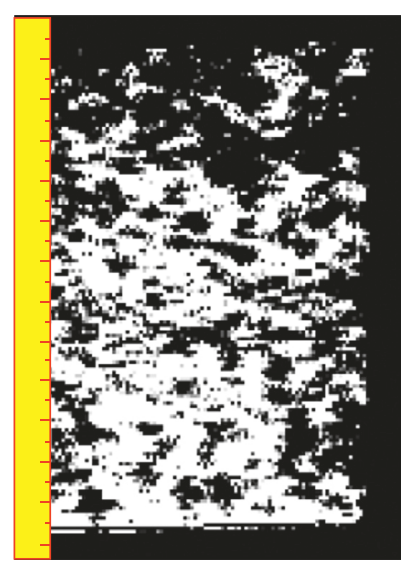

(a)

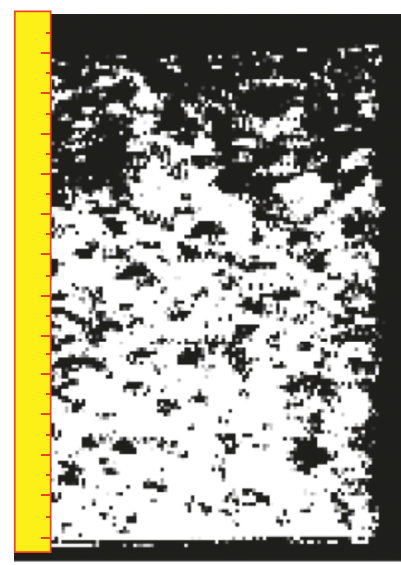

(d)

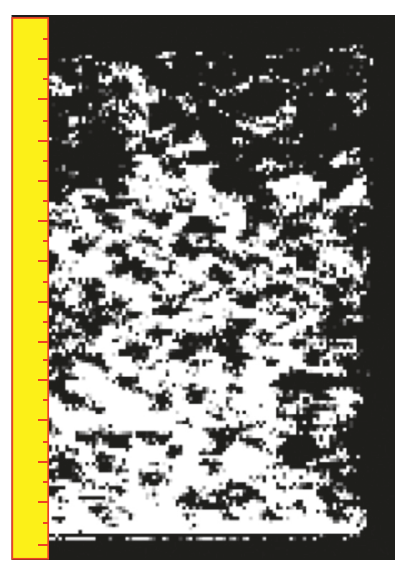

(b)

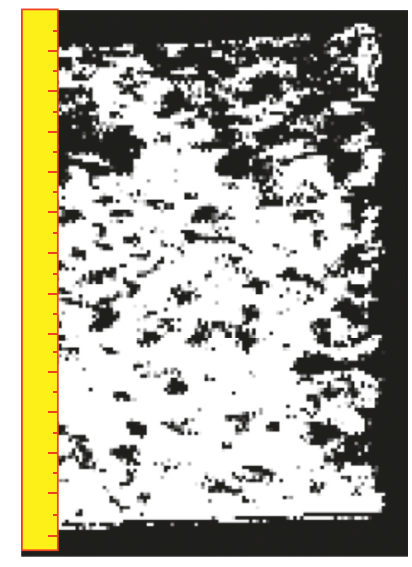

(e)

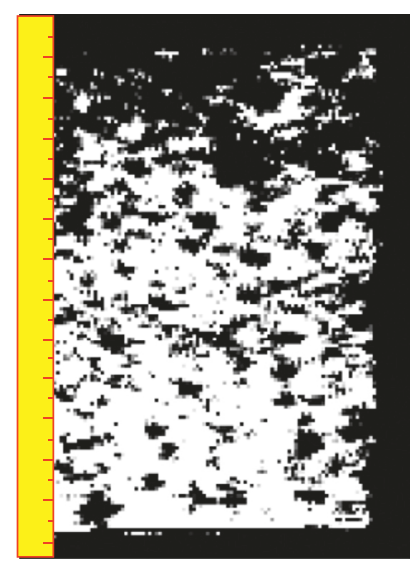

(c)

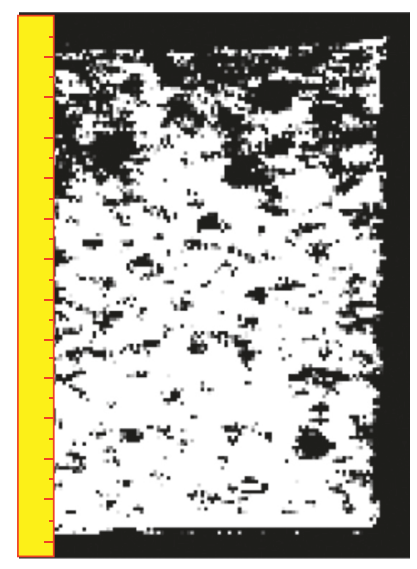

(f)

Figure 11: Phase of pure water leaching. (a) $1 \mathrm{~h}$, (b) $2 \mathrm{~h}$, (c) $3 \mathrm{~h}$, (d) $4 \mathrm{~h}$, (e) $5 \mathrm{~h}$, and (f) $6 \mathrm{~h}$.

area has a banding distribution in the upper part of the sample, but no obvious changes can be observed at the bottom of the sample, compared with the image at the 6th hour, which shows that the reaction occurs inside the sample when pure water is replaced by the $\left(\mathrm{NH}_{4}\right)_{2} \mathrm{SO}_{4}$ solution between the 6 th and 7 th hour. The ion exchange mainly occurs at the top of the sample, and the leaching chemical replacement reaction leads to the change in particle structure, giving rise to the movement and reconstruction of the pore particles and the disappearance of some pores. Pores with large apertures are transformed into pores with small and medium apertures, and the area of ion exchange tends to be more compact. The change becomes more obvious in Figure 9; the black area increases more rapidly in the middle-upper part, which shows that the pore structure is undergoing a rapid change under the effects of ion exchange. After the 9th hour, the black area starts to shift to the lower-middle part. The ion exchange reaction has been completed in the upper part, which again enters into the model of simple liquid seepage. Meanwhile, another obvious change can be observed: the bright area increases again in the middle-upper part, indicating that pores appear in large numbers in the middleupper part. As the apertures increase, it proves that the pore structure under the simple seepage effect tends to be looser. From the 9th hour to 11th hour, the area with the black stripe moves downward, and the bright area above the black area recovers layer by layer. After 12 hours, the stripped black area completely disappears, and the white area is distributed to a great extent. According to Figure 6, the ion exchange reaction has been completed at this point in time, and only the seepage effect influences the micropore structures of orebodies.

The influence of pure water leaching on the pore structure is a seepage effect, while the influences of $\left(\mathrm{NH}_{4}\right)_{2} \mathrm{SO}_{4}$ solution leaching on the micropore structure of an orebody are both ion exchange and seepage effects. The black area moving downward in Figure 12 is completely different from that of Figure 11. Therefore, under the coupling effect of ion exchange and seepage, it is the chemical action of ion exchange, and not the physical action of seepage, that influences the microstructure. Meanwhile, through the contrastive analysis of Figures 11 and 12, the overall manifestation of the micropore structures of rare earth orebodies after $\left(\mathrm{NH}_{4}\right)_{2} \mathrm{SO}_{4}$ solution leaching is that the pore sizes shrink, the total number of pores is reduced, and the pore structure tends to be more compact. 


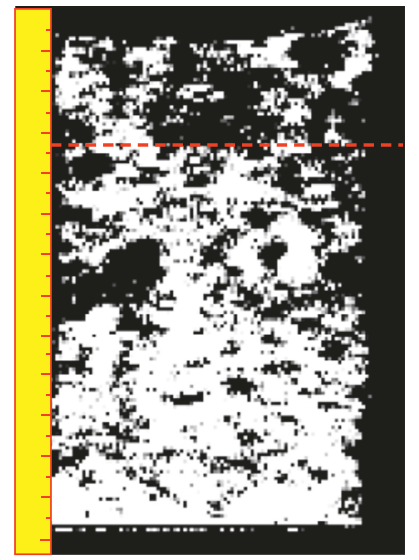

(a)

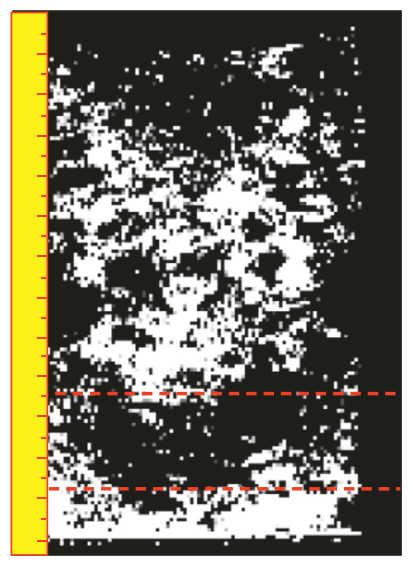

(d)

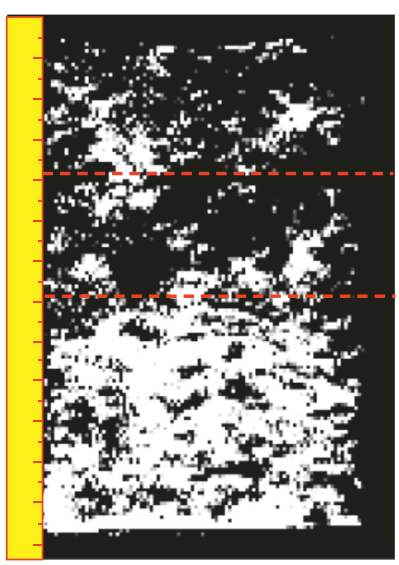

(b)

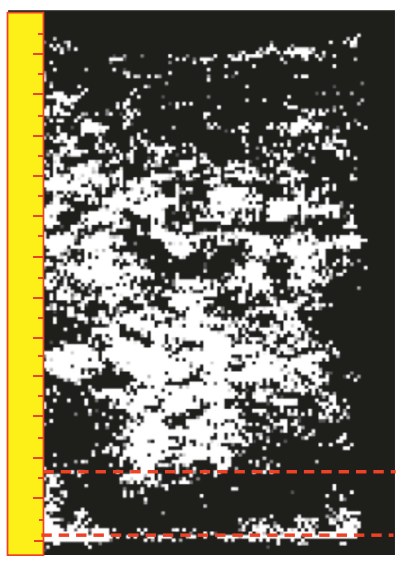

(e)

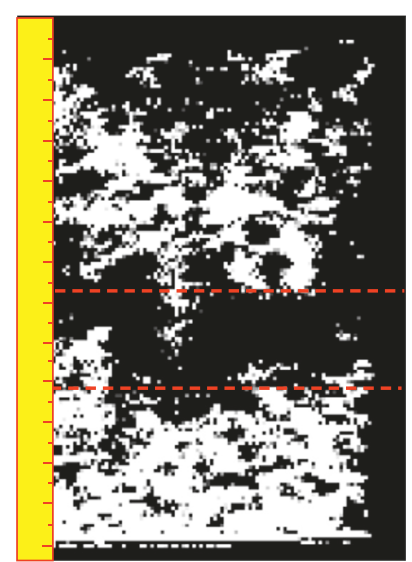

(c)

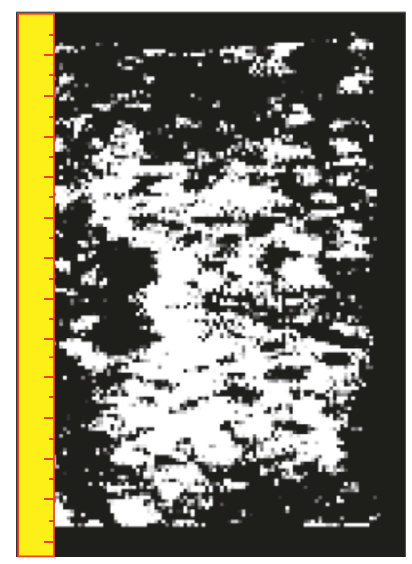

(f)

FIgUre 12: Phase of $\left(\mathrm{NH}_{4}\right)_{2} \mathrm{SO}_{4}$ solution (2.5\%). (a) $7 \mathrm{~h}$, (b) $8 \mathrm{~h}$, (c) $9 \mathrm{~h}$, (d) $10 \mathrm{~h}$, (e) $11 \mathrm{~h}$, and (f) $12 \mathrm{~h}$.

\section{Conclusions}

This paper investigated a leaching experiment on ion-type rare earth orebodies and conducted a contrastive analysis of the leaching results of two different solutions. The following conclusions were obtained:

(1) The pure water leaching process does not involve ion exchange, belonging simply to a liquid seepage process, while the $\left(\mathrm{NH}_{4}\right)_{2} \mathrm{SO}_{4}$ solution leaching process is the coupling of ion exchange and liquid seepage.

(2) In the leaching process, the micropore structures of rare earth orebodies are subjected to the coupling effect of solution seepage and ion exchange. Water leaching leads to an increase of inner pores and apertures. The microstructure also tends to be looser. $\left(\mathrm{NH}_{4}\right)_{2} \mathrm{SO}_{4}$ leaching leads to the movement and reconstruction of particles, decreasing the apertures and the number of pores. The microstructure also tends to be more compact.

(3) In the leaching process, the ion exchange reaction occurs downward, layer by layer, with the solution seepage conforming to the laminar flow law of seepage theory. Under $\left(\mathrm{NH}_{4}\right)_{2} \mathrm{SO}_{4}$ solution leaching, it is the chemical action of ion exchange but not the physical action produced by the seepage effect that influences the microstructure of an orebody. Overall, the chemical replacement reaction of ion-type rare earth leaching possesses certain recovery effects on the microstructure of orebodies.

\section{Conflicts of Interest}

The authors declare that they have no conflicts of interest.

\section{Acknowledgments}

The research was financially supported by National Natural Science Foundation of China (nos. 51304083, 51364011, 51564012, and 51504102), Postdoctoral Science Foundation of China (no. 2014M552562XB), Science and Technology Support Plan of Jiangxi (no. 20141BBE50005), Science and Technology Project Founded by the Education Department of Jiangxi Province (GJJ150653), Qingjiang Excellent Young Talents of Jiangxi University of Science, and Technology and Jiangxi University of Science and Technology Excellent Doctoral Thesis Training Project (YB2017001). 


\section{References}

[1] T. S. Qiu, D. M. Zhu, X. H. Fang, Q. H. Zeng, G. K. Gao, and H. L. Zhu, "Leaching kinetics of ionic rare-earth in ammonianitrogen wastewater system added with impurity inhibitors," Journal of Rare Earths, vol. 32, no. 12, pp. 1175-1183, 2014.

[2] K. Sanematsu, Y. Kon, A. Imai, K. Watanabe, and Y. Watanabe, "Geochemical and mineralogical characteristic of ion-adsorption type REE mineralization in Phuket, Thailand," Mineralium Deposita, vol. 48, no. 4, pp. 437-451, 2013.

[3] L. Zhang, K. X. Wu, L. K. Chen, P. Zhu, and Y. H. Ou, "Overview of metallogenic features of ion-adsorption type REE deposits in Southern Jiangxi Province," Journal of the Chinese Society of Rare Earths, vol. 33, no. 1, p. 10, 2015.

[4] Q. B. Zhang, Y. X. Hua, C. Y. Xu, Y. Li, J. Li, and P. Dong, "Non-haloaluminate ionic liquids for low- temperature electrodeposition of rare-earth metals-a review," Journal of Rare Earths, vol. 33, no. 10, pp. 1017-1025, 2015.

[5] L. Zhao, Z. Dong, G. L. Ma, and W. J. Yuan, "Solution extraction of several lanthanides from nitric acid with isohexyl-BTP in $\left[\mathrm{C}_{\mathrm{n}} \mathrm{mim}\right]\left[\mathrm{NTf}_{2}\right]$ ionic liquid," Journal of Rare Earths, vol. 33, no. 11, pp. 1182-1188, 2015.

[6] X. Q. Sun, Y. Ji, J. Chen, and J. T. Ma, "Solvent impregnated resin prepared using task-specific ionic liquids for rare earth separation," Journal of Rare Earths, vol. 27, no. 6, pp. 932-936, 2009.

[7] J. Dewanckele, T. De Kock, M. A. Boone et al., “4D imaging and quantification of pore structure modifications inside natural building stones by means of high resolution X-ray CT," Science of the Total Environment, vol. 416, no. 2, pp. 436-448, 2012.

[8] J. R. Kyle and R. A. Ketcham, "Application of high resolution $\mathrm{X}$-ray computed tomography to mineral deposit origin, evaluation, and processing," Ore Geology Reviews, vol. 65, no. 4, pp. 821-839, 2015.

[9] L. J. Munkholm, R. J. Heck, and B. Deen, "Soil pore characteristics assessed from X-ray micro-CT derived images and correlations to soil friability," Geoderma, vol. 181-182, no. 7, pp. 22-29, 2012.

[10] P. Kodali, N. Dhawan, T. Depci et al., "Particle damage and exposure analysis in HPGR crushing of selected copper ores for column leaching," Minerals Engineering, vol. 24, no. 13, pp. 1478-1487, 2011.

[11] A. Nosrati, K. Quast, D. F. Xu et al., "Agglomeration and column leaching behaviour of nickel laterite ores: effect of ore mineralogy and particle size distribution," Hydrometallurgy, vol. 146, no. 5, pp. 29-39, 2014.

[12] X Q. Yan, Y. G. Fang, and P. Zhang, "Experiment study on the effects of bentonite on the micropore structure characteristics of soil," Chinese Journal of Geotechnical Engineering, vol. 33, no. 8, pp. 1302-1307, 2011.

[13] D. S. Cui, W. Xiang, Q. Chen, Q. B. Liu, L. Z. Lang, and Y. P. Wu, "Pore characteristics of sliding zone soils of Huangtupo landslide by vacuum freeze-dried methods," Earth Science-Journal of China University of Geosciences, vol. 39, no. 10, p. 1531, 2014.

[14] X. Li, L. M. Zhang, and G. D. Ao, "Variations of pore structure, void ratio, and water content in soil drying process," Rock and Soil Mechanics, vol. 32, no. S1, pp. 100-105, 2011.

[15] G. Q. Deng and Y. M. Yang, "A review of the mining technologies of ion-absorbed rare earth mineral," Chinese Rare Earths, vol. 37, no. 3, p. 129, 2016.

[16] R. A. Chi, J. Tian, X. P. Luo, Z. G. Xu, and Z. Y. He, "The basic research on the weathered crust elution-deposited rare earth ores," Nonferrous Metals Science and Engineering, vol. 3, no. 4, p. 1, 2012.

[17] K. P. Zhou, J. L. Li, Y. J. Xu et al., "Experimental study of NMR characteristics in rock under freezing and thawing cycles," Chinese Journal of Rock Mechanics and Engineering, vol. 31, no. 4, p. 731, 2012.

[18] X. J. Li, S. D. Nie, Y. J. Wang, and P. Q. Yang, “An improved two-dimensional inversion algorithm for low-field NMR diffusion-transverse relaxation data," Chinese Journal of Magnetic Resonance, vol. 30, no. 3, p. 322, 2013.

[19] K. W. Wang and N. Li, "Numerical simulation on effects of reservoir characteristics and saturation on T2 spectra of nuclear magnetic resonance," Acta Petrolei Sinica, vol. 30, no. 3, p. 422, 2009.

[20] S. T. Bai, D. J. Cheng, J. B. Wan et al., "Quantitative characterization of sandstone NMR T2 spectrum," Acta Petrolei Sinica, vol. 27, no. 3, pp. 382-391, 2016. 

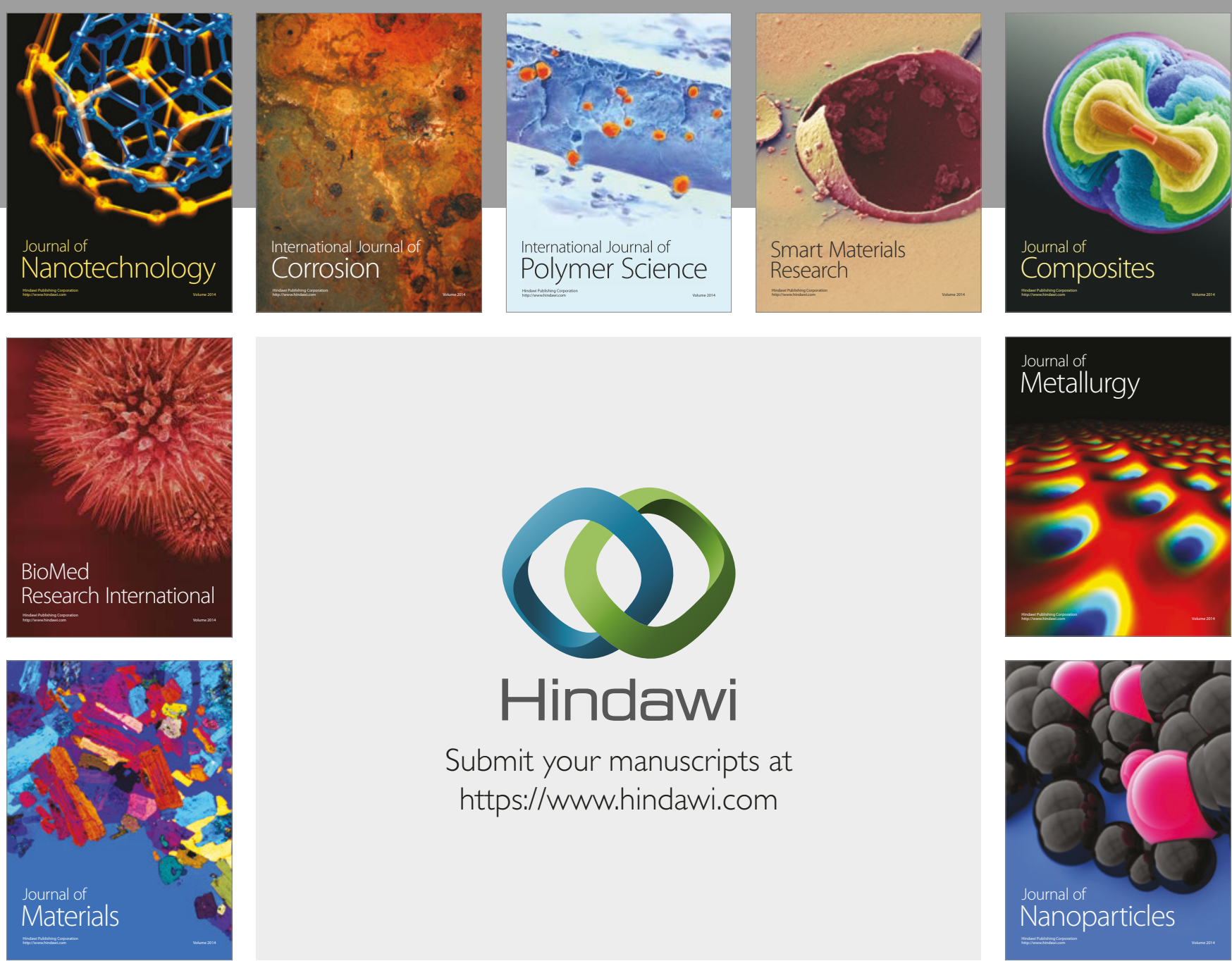

\section{Hindawi}

Submit your manuscripts at

https://www.hindawi.com
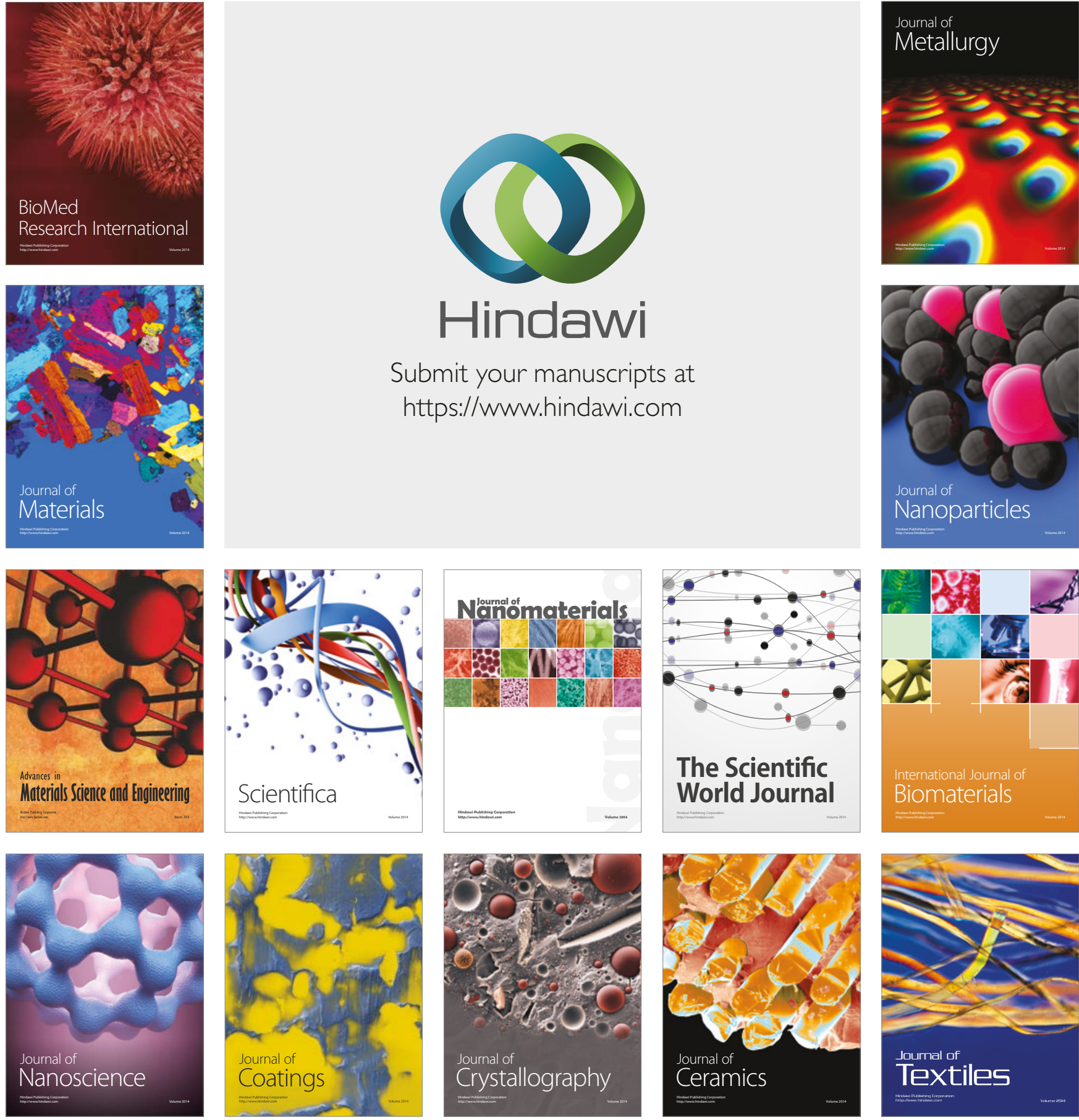

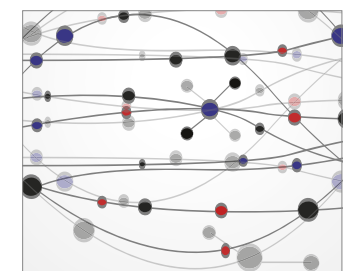

The Scientific World Journal
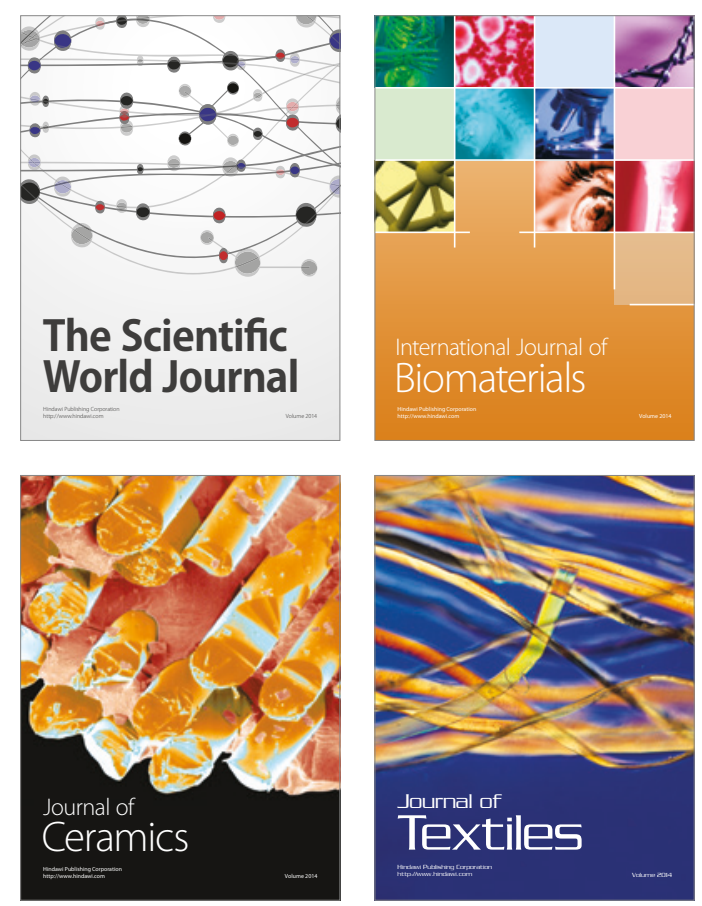